Article

\title{
Method for Remote Determination of Object Coordinates in Space Based on Exact Analytical Solution of Hyperbolic Equations
}

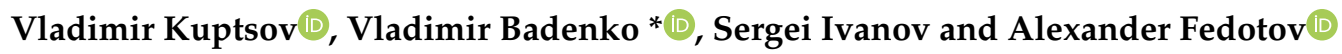 \\ Institute of Physics, Nanotechnology and Telecommunications, Peter the Great Saint-Petersburg Polytechnic \\ University, 29 Polytechnicheskaya str., 195251 Saint Petersburg, Russia; vdkuptsov@yandex.ru (V.K.); \\ ivanov_si@spbstu.ru (S.I.); afedotov@spbstu.ru (A.F.) \\ * Correspondence: badenko_vl@spbstu.ru; Tel.: +7-921-309-41-00
}

Received: 4 August 2020; Accepted: 21 September 2020; Published: 24 September 2020

check for updates

\begin{abstract}
Accurate remote determination of the object coordinates in 3D space is one of the main questions in many applications. In one of the most popular methods, such determination of the location of an object uses the measurement by receiving an electromagnetic signal transmitted by several spatially distributed base stations (BS). The main problem is that it is necessary to reduce errors and computation time. To overcome these difficulties, an analytical method for determining the position of an object based on the analysis of time difference of arrival (TDoA) of signals from the transmitter of the object to the receivers of the BS is proposed. One of the main advantages of this method is that it is possible to eliminate the ambiguity in determining the coordinates of the object in space and to increase the accuracy of determining the coordinates when the TDoA measurement between base stations fluctuates. Applications for autonomous automotive vehicles and space-based positioning systems are analyzed. The results obtained show that the proposed algorithm has an accuracy of determining coordinates several times higher than the method of linearization of hyperbolic equations and is less sensitive to TDoA fluctuations at base stations.
\end{abstract}

Keywords: positioning; unmanned aerial vehicle; time difference of arrival; ambiguity; determination of 3D coordinates; desynchronization in time; TDoA fluctuations; local positioning system (LPS); global positioning system (GPS)

\section{Introduction}

Many modern applications need accurate 2D/3D object coordinates, which are determined by remote sensing methods. These applications include, for example, driving unmanned vehicles or security systems in "smart city" projects [1,2]. In one of the most popular methods, such determination of the location of an object uses measurements by receiving an electromagnetic signal transmitted by several spatially distributed base stations, taking into account shapes and other properties of objects [3]. Commonly used measurements are time delay for active scenarios [4] and time differences for passive environments [5]. The main aim of positioning tasks is to determine the coordinates (position) of the object/target, which can be carried out using various algorithms $[6,7]$ and the most popular of them are discussed as follows.

For example, in [8], a control algorithm for an unmanned aerial vehicle (UAV) to circumnavigate an unknown target at a fixed radius when the UAV is unable to determine its location based on measurement errors and modeling the system by stochastic differential equations. Among other algorithms, it can be pointed out that with RSSI (Received Strength Signal Indication), the distance to the object is estimated by the signal power, but this algorithm usually has successful application for 
indoor positioning with a wireless network [9-11]. The algorithm AoA (Angle of Arrival) is based on the location of the object by determination within the triangle area formed by the intersection of the axes of the antenna patterns of the sectors of the three base stations (modified triangulation method) and usually plays a role as a part of another target tracking algorithm, for example, 3D pseudolinear Kalman filter (KF) algorithm [12-14] or 3D source localization method for acoustic sensor networks [15]. The algorithm ToF (Time of Flight) is based on measuring the travel time of an electromagnetic wave from an object transmitter to a base station by using a signal with linear frequency modulation [16-18]. This algorithm is very popular for applications in medicine [19] but also has application in unmanned aerial vehicle navigation [20]. Close in meaning to ToF is the algorithm ToA (Time of Arrival), which is based on distance calculation by measuring the signal transit time from the mobile terminal to the base station as the difference between the time the signal was sent and received [21-24]. The algorithm ToA is often used with TDoA (Time Difference of Arrival) [25] and FDoA (Frequency difference of arrival) [26], which are also often used together [27-30]. TDoA is a very popular algorithm for different applications and measures the difference in the time of arrival of a signal from an object to several base stations [31-33]. FDoA, which is also named DD (Differential Doppler), is a method analogous to TDoA for estimating the location of a radio emitter based on observations from other points according to different Doppler shift observations of the emitter at different locations [34,35]. Very similar to ToA is RTT (Round Trip Time) method, in which a base station also sends a signal to a mobile device and waits for a response signal [36-38].

The TDoA method is highly accurate, and one of its main features is that it requires time synchronization only at base stations [39-43]. If one analyzes the surfaces on which the difference in the arrival times of the microwave signal in TDoA method to two base stations (BS) is constant, one finds that they are hyperboloids in space with both BSs in their focus $[39,44]$. In such cases, the coordinates of the target can be identified as an intersection of these surfaces using, for example, the Taylor-series method at a reasonable noise level [45-47]. But this Taylor-series method is an iterative method, and it strongly depends on an initial assumption (starting point) and requires large computational resources [48]. For solving such problems in [47], it was proposed to use Taylor series expansion for linearization of hyperbolic equations, which leads to a more simple matrix form of equations $[49,50]$.

A linear frequency-modulated microwave radar waveform is used in many applications, including microwave-range automobile radars for the simultaneous measurement of velocity and distance, which use signals of a special shape, including frequency-modulated continuous-wave (FMCW) [51]. Mathematical models of the received microwave signal for automotive radar systems taking into account a different range of velocity and distance for vehicles, were proposed in [52-58]. In [52], it is shown that the use of phase methods to determine the speed of the target significantly reduces the noise immunity of the radar, and methods based on processing the amplitude spectrum of the received radio signal by the Fast Fourier Transformation (FFT) are therefore preferable. In [53] is the algorithm for estimating the speed and distance to the target as applied to the automotive radar with linear FMCW, using the FFT for amplitude spectrum and taking into account the occurrence of false targets. The results of synthesis and analysis of the effectiveness of the optimal location-based system for joint detection and estimation of informative parameters of quasi-determined radar signals with frequency modulated continuous wave are presented in [56]. In [57], the authors explore the properties of the multi-target method for small unmanned vehicle parameters' remote determination by microwave radars, which allows significantly expanding the range of unambiguously determined speeds of unmanned vehicles. The reasons for the occurrence of false targets and their number in the mode of the parameters simultaneous measurement for the set of unmanned vehicles are identified. Using a computer experiment in LabVIEW (Laboratory Virtual Instrumentation Engineering Workbench), the probability of the unmanned vehicles' true parameters determination at various ratios of the signal to noise at the input of the radar receiver in multi-target mode was investigated. The influence of millimeter-wave radar receiver noise on the probability of unambiguous determination of unmanned vehicles' speed and range in the intelligent transportation system of the "smart city" is 
investigated in [58]. For the proposed new multi-target detection method for FMCW radar, the effect of the technical parameters of the vehicle radars on the required signal-to-noise ratio (SNR) of the receiver is estimated to ensure the probability of true determination of target parameters at $98 \%$.

An exact analytical solution of equations for the task of localization of an object using a number of sensors is often challenged by outlier observations when the number of TDoA measurements is equal to the number of unknown transmitter coordinates [59-61]. The measurement equations are nonlinear, and an exact algebraic solution exists only in the specific case of a predetermined scenario in which the number of measurements is equal to the number of unknowns. The solution in [60] cannot use TDoA measurements with additional base stations improving positioning accuracy, and it has a high computational requirement for solving nonlinear equations. Compared to the solutions in [60], the solution proposed in [61] is more computationally attractive, requires only the roots of a quadratic equation, is more general and robust, and can work with arbitrary sensor arrangements.

A method proposed in [62] is based on Spherical-Intersection using only the root of a quadratic equation, but this method is not robust and fails to produce a reasonable solution for some sensor arrangements, due to the need to invert a matrix with the coordinates of sensors. Exact solutions can also be found in [63-65], and authors sometimes formulated an appropriated optimization task in form of a polynomial system, the solution of which was found by numerical algebraic methods or polynomial continuation techniques.

A good description of analytical methods is presented in [66-68]. Transformation of the coordinates of a hyperbola during a shift and rotation on a plane can be found in [66]. According to this transformation in [67], the effectiveness of two methods of TDOA analytical solution was compared based on the task of finding intersection points of hyperboloids (possible positions of a target). The first method analyzed was based on coordinate transformation from the initial system to a new system to simplify equations solving, and the second one was based on matrix. In [68], the results of an experimental verification of an analytical method based on coordinate transformation are presented. Finally, in [69], a high-precision analytical TDoA algorithm for determining the coordinates of an object on a plane (2D model) is proposed for eliminating the ambiguity of coordinates determination. Of great interest are recent works especially for indoor and AoA localization [70-73].

In this paper, an analytical method for determining the position of a target based on the analysis of TDoA of microwave radar signals from the transmitter to Base Stations (BS) receivers is proposed. The main feature of the method is that it can eliminate the ambiguity in determining of 3D coordinates of a target and improves the accuracy of determining coordinates when the TDoA measurements fluctuate on BS.

The materials of the article are presented in the following form: In Section 2.1, the fundamentals of the linearization method are presented without going into the specifics of solving linear equations by matrix methods. Section 2.2 sets out the proposed analytical method for determining the coordinates of an object in a positioning system. Section 3.1 describes the spatial ambiguity problem inherent in simple analytical methods. A comparison of the accuracy of determining coordinates by the linearization method and the proposed method at various levels of TDoA fluctuations for local and global positioning systems are carried out in Section 3.2. The results are discussed in Section 4. The paper ends with conclusions (Section 5) from the work done.

\section{Materials and Methods}

\subsection{Linearization Method of Hyperbolic Equations}

The method of linearizing hyperbolic equations was first proposed in [47]. The widespread use of this method at the present time is explained by the fact that it allows one to avoid solving nonlinear equations, which means that significant computing power of the positioning system processor is not required. The method consists in transforming nonlinear equations into a set of linear equations $\mathrm{Ax}=\mathrm{b}$ in matrix form and further solving the system of equations by matrix methods $[25,46,47,50,61,65]$. 
We will compare our proposed algorithm (Section 2.2) with the linearization method; therefore, we will briefly outline the basics of the linearization method without delving into the specifics of solving linear equations by matrix methods.

In the absence of errors in measuring TDoA and receiving line-of-sight (LOS) signals, the real values of the difference in the arrival times of the TDoA signal between $\mathrm{BS}_{i}$ and $\mathrm{BS}_{1}$ are determined by the expression:

$$
\Delta \tau_{i 1}=\frac{r_{i}-r_{1}}{c_{l}}=\frac{r_{i 1}}{c_{l}}, i=2, \ldots, M
$$

where $M$-amount of BS, $c_{l}$-speed of light, $r_{i}=\sqrt{\left(x_{i}-x\right)^{2}+\left(y_{i}-y\right)^{2}+\left(z_{i}-z\right)^{2}}$-distance between object and $\mathrm{BS}_{i}, r_{1}$-distance between object and $\mathrm{BS}_{1},\left[x_{i}, y_{i}, z_{i}\right]$ - $\mathrm{BS}_{i}$ coordinates, $[x, y, z]$-object coordinates. From $r_{i 1}=r_{i}-r_{1}$ follows $r_{i 1}+r_{1}=r_{i}$. Substitution of the coordinates of the Cartesian system in Equation (1), squaring the right and left sides of the equation, expansion in a Taylor series, and linearization leads to the equation:

$$
\begin{gathered}
\left(x_{i}-x_{1}\right)\left(x-x_{1}\right)+\left(y_{i}-y_{1}\right)\left(y-y_{1}\right)+\left(z_{i}-z_{1}\right)\left(z-z_{1}\right)+c_{l} \cdot \Delta \tau_{i 1} \cdot r_{1}=\ldots \\
\ldots=\frac{1}{2}\left(\left(x_{i}-x_{1}\right)^{2}+\left(y_{i}-y_{1}\right)^{2}+\left(z_{i}-z_{1}\right)^{2}-\left(c_{l} \cdot \Delta \tau_{i 1}\right)^{2}\right)
\end{gathered}
$$

which in matrix form has the form $A \boldsymbol{\theta}=\boldsymbol{b}$, where

$$
\begin{aligned}
& \boldsymbol{A}=\left[\begin{array}{cccc}
x_{2}-x_{1} & y_{2}-y_{1} & z_{2}-z_{1} & c_{l} \cdot \Delta \tau_{21} \\
x_{3}-x_{1} & y_{3}-y_{1} & z_{3}-z_{1} & c_{l} \cdot \Delta \tau_{31} \\
\cdot & \cdot & \cdot & \cdot \\
x_{M}-x_{1} & y_{M}-y_{1} & z_{M}-z_{1} & c_{l} \cdot \Delta \tau_{M 1}
\end{array}\right], \boldsymbol{\theta}=\left[\begin{array}{llll}
x-x_{1} & y-y_{1} & z-z_{1} & r_{1}
\end{array}\right]^{T} \\
& \boldsymbol{b}=\frac{1}{2}\left[\begin{array}{c}
\left(x_{2}-x_{1}\right)^{2}+\left(y_{2}-y_{1}\right)^{2}+\left(z_{2}-z_{1}\right)^{2}-\left(c_{l} \cdot \Delta \tau_{21}\right)^{2} \\
\left(x_{3}-x_{1}\right)^{2}+\left(y_{3}-y_{1}\right)^{2}+\left(z_{3}-z_{1}\right)^{2}-\left(c_{l} \cdot \Delta \tau_{31}\right)^{2} \\
\cdot \\
\left(x_{M}-x_{1}\right)^{2}+\left(y_{M}-y_{1}\right)^{2}+\left(z_{M}-z_{1}\right)^{2}-\left(c_{l} \cdot \Delta \tau_{M 1}\right)^{2}
\end{array}\right]
\end{aligned}
$$

The superscript [ $]^{T}$ denotes a matrix transposition operation. The solution of the equation $\boldsymbol{A \theta}$ $=\boldsymbol{b}$ can be carried out in various ways of solving the system of linear equations. After calculating the values of the vector $\boldsymbol{\theta}$, the coordinates of the target are determined $x=\theta_{1}+x_{1} ; y=\theta_{2}+y_{1}$; $z=\theta_{3}+z_{1}$; where $x_{1}, y_{1}, z_{1}$ - known coordinates of $\mathrm{BS}_{1}$.

\subsection{The Proposed Analytical Method for Determining the Coordinates of an Object in a Positioning System with 5 Base Stations}

The variant of building a positioning system in space with 4 BSs is the most economically profitable; however, it has a disadvantage that the intersection of two hyperboloids can occur along two lines in space. In this case, it becomes impossible to identify the true position of the object. To eliminate the ambiguity in determining the position of the object, it is necessary to include the fifth BS in the positioning system. With the addition of the fifth BS, it becomes possible to solve four systems of equations, which will have one common root corresponding to the true position of the target. An analytical solution to the problem of determining the location of an object in 3D for a positioning system of 4 BSs was obtained in [67], but in [67], not all values of the coefficients are given, which complicates the direct use of the algorithm. In addition, in [67], the problem of eliminating ambiguity in determining the coordinates was not solved.

In what follows, the development of an analytical algorithm that eliminates the spatial zones of ambiguity in determining the coordinates of the target in space with high accuracy coordinates determination of the object is presented.

Let us consider a spatial positioning system that includes five microwave signal receivers $\mathrm{BC}_{C}$, $\mathrm{BS}_{L}, \mathrm{BS}_{R}, \mathrm{BS}_{U}, \mathrm{BS}_{D}$ (Figure 1). 


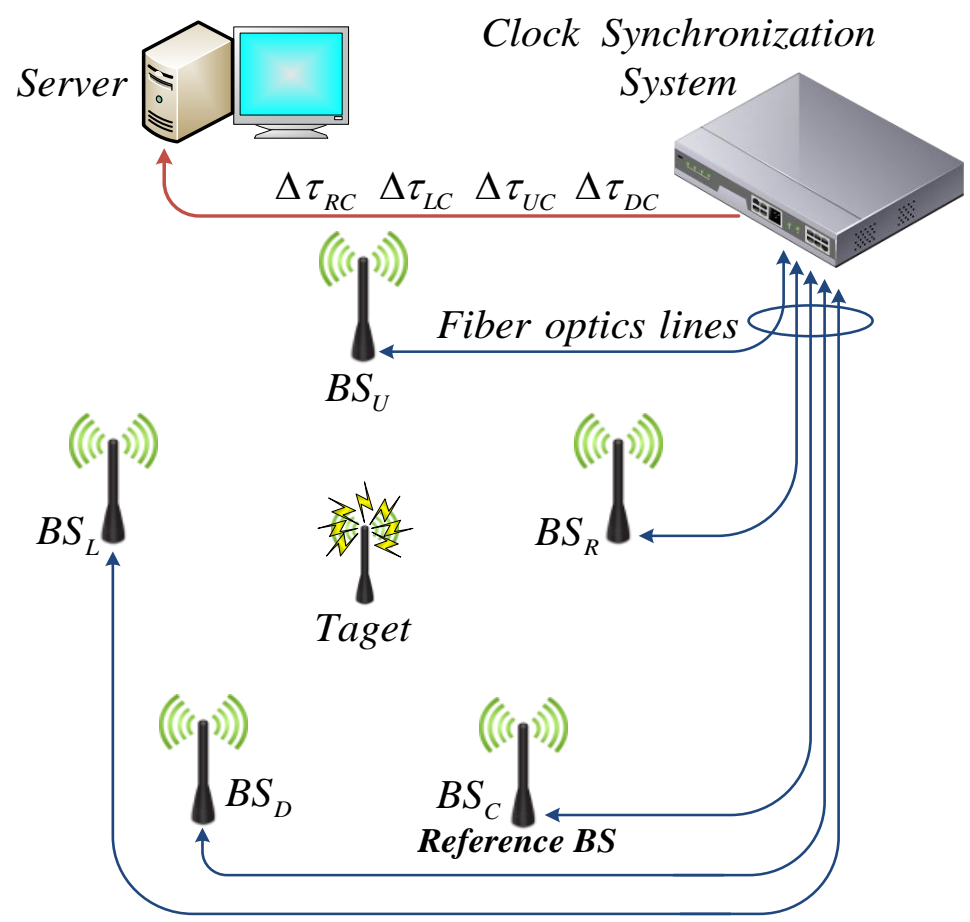

Figure 1. Block diagram of the time difference of arrival (TDoA)-based positioning system with 5 base stations (BSs).

The coordinates of the transmitter position are solutions to the system of hyperbolic equations:

$$
\left\{\begin{array}{l}
\sqrt{\left(x-x_{L}\right)^{2}+\left(y-y_{L}\right)^{2}+\left(z-z_{L}\right)^{2}}-\sqrt{\left(x-x_{C}\right)^{2}+\left(y-y_{C}\right)^{2}+\left(z-z_{C}\right)^{2}}=c \cdot \Delta \tau_{L C} \\
\sqrt{\left(x-x_{R}\right)^{2}+\left(y-y_{R}\right)^{2}+\left(z-z_{R}\right)^{2}}-\sqrt{\left(x-x_{C}\right)^{2}+\left(y-y_{C}\right)^{2}+\left(z-z_{C}\right)^{2}}=c \cdot \Delta \tau_{R C} \\
\sqrt{\left(x-x_{U}\right)^{2}+\left(y-y_{U}\right)^{2}+\left(z-z_{U}\right)^{2}}-\sqrt{\left(x-x_{C}\right)^{2}+\left(y-y_{C}\right)^{2}+\left(z-z_{C}\right)^{2}}=c \cdot \Delta \tau_{U C} \\
\sqrt{\left(x-x_{D}\right)^{2}+\left(y-y_{D}\right)^{2}+\left(z-z_{D}\right)^{2}}-\sqrt{\left(x-x_{C}\right)^{2}+\left(y-y_{C}\right)^{2}+\left(z-z_{C}\right)^{2}}=c \cdot \Delta \tau_{D C}
\end{array}\right.
$$

where $\Delta \tau_{L C}, \Delta \tau_{R C}, \Delta \tau_{U C}, \Delta \tau_{D C}$ - TDoA of the microwave signal between $B S_{L, R, U, D}$ and reference station $\mathrm{BS}_{C}$.

After substitution of $X=x-x_{C}, Y=y-y_{C}, Z=z-z_{C}$ the system of equations can be rewritten as:

$$
\left\{\begin{array}{c}
\sqrt{\left(X-X_{L}\right)^{2}+\left(Y-Y_{L}\right)^{2}+\left(Z-Z_{L}\right)^{2}}-\sqrt{X^{2}+Y^{2}+Z^{2}}=L \\
\sqrt{\left(X-X_{R}\right)^{2}+\left(Y-Y_{R}\right)^{2}+\left(Z-Z_{R}\right)^{2}}-\sqrt{X^{2}+Y^{2}+Z^{2}}=R \\
\sqrt{\left(X-X_{U}\right)^{2}+\left(Y-Y_{U}\right)^{2}+\left(Z-Z_{U}\right)^{2}}-\sqrt{X^{2}+Y^{2}+Z^{2}}=U \\
\sqrt{\left(X-X_{D}\right)^{2}+\left(Y-Y_{D}\right)^{2}+\left(Z-Z_{D}\right)^{2}}-\sqrt{X^{2}+Y^{2}+Z^{2}}=D \\
X_{L}=x_{L}-x_{C}, Y_{L}=y_{L}-y_{C}, Z_{L}=z_{L}-z_{C}, L=c \cdot \Delta \tau_{L C} \\
X_{R}=x_{R}-x_{C}, Y_{R}=y_{R}-y_{C}, Z_{R}=z_{R}-z_{C}, R=c \cdot \Delta \tau_{R C} \\
X_{U}=x_{U}-x_{C}, Y_{U}=y_{U}-y_{C}, Z_{U}=z_{U}-z_{C}, U=c \cdot \Delta \tau_{U C} \\
X_{D}=x_{D}-x_{C}, Y_{D}=y_{D}-y_{C}, Z_{D}=z_{D}-z_{C}, D=c \cdot \Delta \tau_{D C}
\end{array}\right.
$$

Let us denote $K=\sqrt{X^{2}+Y^{2}+Z^{2}}$, where $K>0$ :

$$
K^{2}=X^{2}+Y^{2}+Z^{2}
$$


The system of Equations (5) can be rewritten as:

$$
\left\{\begin{array}{l}
\sqrt{\left(X-X_{L}\right)^{2}+\left(Y-Y_{L}\right)^{2}+\left(Z-Z_{L}\right)^{2}}=K+L \\
\sqrt{\left(X-X_{R}\right)^{2}+\left(Y-Y_{R}\right)^{2}+\left(Z-Z_{R}\right)^{2}}=K+R \\
\sqrt{\left(X-X_{U}\right)^{2}+\left(Y-Y_{U}\right)^{2}+\left(Z-Z_{U}\right)^{2}}=K+U \\
\sqrt{\left(X-X_{D}\right)^{2}+\left(Y-Y_{D}\right)^{2}+\left(Z-Z_{D}\right)^{2}}=K+D
\end{array}\right.
$$

Squaring and reducing the general terms leads to the form:

$$
\begin{aligned}
& \left\{\begin{array}{rl}
-2 X_{L} X-2 Y_{L} Y-2 Z_{L} Z & =2 L K+L^{2}-X_{L}^{2}-Y_{L}^{2}-Z_{L}^{2} \\
-2 X_{R} X-2 Y_{R} Y-2 Z_{R} Z & =2 R K+R^{2}-X_{R}^{2}-Y_{R}^{2}-Z_{R}^{2} \\
-2 X_{U} X-2 Y_{U} Y-2 Z_{U} Z & =2 U K+U^{2}-X_{U}^{2}-Y_{U}^{2}-Z_{U}^{2} \\
-2 X_{D} X-2 Y_{D} Y-2 Z_{D} Z & =2 D K+D^{2}-X_{D}^{2}-Y_{D}^{2}-Z_{D}^{2}
\end{array},\right. \text { or } \\
& \left\{\begin{array}{rl}
-2 X_{L} X-2 Y_{L} Y-2 Z_{L} Z & =E+2 L K \\
-2 X_{R} X-2 Y_{R} Y-2 Z_{R} Z & =F+2 R K \\
-2 X_{U} X-2 Y_{U} Y-2 Z_{U} Z & =G+2 U K \\
-2 X_{D} X-2 Y_{D} Y-2 Z_{D} Z & =H+2 D K
\end{array},\right.
\end{aligned}
$$

where $E=L^{2}-X_{L}^{2}-Y_{L}^{2}-Z_{L}^{2} ; F=R^{2}-X_{R}^{2}-Y_{R}^{2}-Z_{R}^{2} ; G=U^{2}-X_{U}^{2}-Y_{U}^{2}-Z_{U}^{2} ; H=D^{2}-X_{D}^{2}-Y_{D}^{2}-Z_{D}^{2}$. In matrix form, the four systems of equations are:

$$
\begin{aligned}
& {\left[\begin{array}{lll}
-2 X_{L} & -2 Y_{L} & -2 Z_{L} \\
-2 X_{R} & -2 Y_{R} & -2 Z_{R} \\
-2 X_{U} & -2 Y_{U} & -2 Z_{U}
\end{array}\right] \cdot\left[\begin{array}{c}
X \\
Y \\
Z
\end{array}\right]=\left[\begin{array}{c}
E+2 L K \\
F+2 R K \\
G+2 U K
\end{array}\right],} \\
& {\left[\begin{array}{lll}
-2 X_{R} & -2 Y_{R} & -2 Z_{R} \\
-2 X_{U} & -2 Y_{U} & -2 Z_{U} \\
-2 X_{D} & -2 Y_{D} & -2 Z_{D}
\end{array}\right] \cdot\left[\begin{array}{c}
X \\
Y \\
Z
\end{array}\right]=\left[\begin{array}{c}
F+2 R K \\
G+2 U K \\
H+2 D K
\end{array}\right],} \\
& {\left[\begin{array}{lll}
-2 X_{U} & -2 Y_{U} & -2 Z_{U} \\
-2 X_{D} & -2 Y_{D} & -2 Z_{D} \\
-2 X_{L} & -2 Y_{L} & -2 Z_{L}
\end{array}\right] \cdot\left[\begin{array}{c}
X \\
Y \\
Z
\end{array}\right]=\left[\begin{array}{c}
G+2 U K \\
H+2 D K \\
E+2 L K
\end{array}\right],} \\
& {\left[\begin{array}{lll}
-2 X_{D} & -2 Y_{D} & -2 Z_{D} \\
-2 X_{L} & -2 Y_{L} & -2 Z_{L} \\
-2 X_{R} & -2 Y_{R} & -2 Z_{R}
\end{array}\right] \cdot\left[\begin{array}{c}
X \\
Y \\
Z
\end{array}\right]=\left[\begin{array}{c}
H+2 D K \\
E+2 L K \\
F+2 R K
\end{array}\right],}
\end{aligned}
$$

It should be noted that when using 4 BSs in the positioning system, there will be only one system of equations.

Solution of the first system of equations $(i=1)$ is:

$$
\begin{gathered}
X_{i}=\frac{1}{\Delta_{i}} \cdot\left|\begin{array}{lll}
E+2 L K & -2 Y_{L} & -2 Z_{L} \\
F+2 R K & -2 Y_{R} & -2 Z_{R} \\
G+2 U K & -2 Y_{U} & -2 Z_{U}
\end{array}\right|=M_{i X} \cdot K+N_{i X}, \text { where } \\
M_{i X}=\frac{2}{\Delta_{i}} \cdot\left(L \Delta_{i X 1}-R \Delta_{i X 2}+U \Delta_{i X 3}\right), N_{i X}=\frac{1}{\Delta_{i}} \cdot\left(E \Delta_{i X 1}-F \Delta_{i X 2}+G \Delta_{i X 3}\right) ; \\
\Delta_{i X 1}=\left|\begin{array}{ll}
-2 Y_{R} & -2 Z_{R} \\
-2 Y_{U} & -2 Z_{U}
\end{array}\right|=4\left(Y_{R} Z_{U}-Y_{U} Z_{R}\right), \\
\Delta_{i X 2}=\left|\begin{array}{ll}
-2 Y_{L} & -2 Z_{L} \\
-2 Y_{U} & -2 Z_{U}
\end{array}\right|=4\left(Y_{L} Z_{U}-Y_{U} Z_{L}\right), \\
\Delta_{i X 3}=\left|\begin{array}{ll}
-2 Y_{L} & -2 Z_{L} \\
-2 Y_{R} & -2 Z_{R}
\end{array}\right|=4\left(Y_{L} Z_{R}-Y_{R} Z_{L}\right)
\end{gathered}
$$




$$
\begin{aligned}
& Y_{i}=\frac{1}{\Delta_{i}} \cdot\left|\begin{array}{lll}
-2 X_{L} & E+2 L K & -2 Z_{L} \\
-2 X_{R} & F+2 R K & -2 Z_{R} \\
-2 X_{U} & G+2 U K & -2 Z_{U}
\end{array}\right|=M_{i Y} \cdot K+N_{i Y} \text {, where } \\
& M_{i \Upsilon}=\frac{2}{\Delta_{i}} \cdot\left(-L \Delta_{i \curlyvee 1}+R \Delta_{i \curlyvee 2}-U \Delta_{i \curlyvee 3}\right), N_{i \Upsilon}=\frac{1}{\Delta_{i}} \cdot\left(-E \Delta_{i \curlyvee 1}+F \Delta_{i \curlyvee 2}-G \Delta_{i \curlyvee 3}\right) \text {; } \\
& \Delta_{i \curlyvee 1}=\left|\begin{array}{ll}
-2 X_{R} & -2 Z_{R} \\
-2 X_{U} & -2 Z_{U}
\end{array}\right|=4\left(X_{R} Z_{U}-X_{U} Z_{R}\right), \\
& \Delta_{i \curlyvee 2}=\left|\begin{array}{ll}
-2 X_{L} & -2 Z_{L} \\
-2 X_{U} & -2 Z_{U}
\end{array}\right|=4\left(X_{L} Z_{U}-X_{U} Z_{L}\right) \\
& \Delta_{i \Upsilon 3}=\left|\begin{array}{ll}
-2 X_{L} & -2 Z_{L} \\
-2 X_{R} & -2 Z_{R}
\end{array}\right|=4\left(X_{L} Z_{R}-X_{R} Z_{L}\right) \\
& Z_{i}=\frac{1}{\Delta_{i}} \cdot\left|\begin{array}{lll}
-2 X_{L} & -2 Y_{L} & E+2 L K \\
-2 X_{R} & -2 Y_{R} & F+2 R K \\
-2 X_{U} & -2 Y_{U} & G+2 U K
\end{array}\right|=M_{i Z} \cdot K+N_{i Z} \text {, where } \\
& M_{i Z}=\frac{2}{\Delta_{i}} \cdot\left(L \Delta_{i Z 1}-R \Delta_{i Z 2}+U \Delta_{i Z 3}\right), N_{i Z}=\frac{1}{\Delta_{i}} \cdot\left(E \Delta_{i Z 1}-F \Delta_{i Z 2}+G \Delta_{i Z 3}\right) \text {; } \\
& \Delta_{i Z 1}=\left|\begin{array}{ll}
-2 X_{R} & -2 Y_{R} \\
-2 X_{U} & -2 Y_{U}
\end{array}\right|=4\left(X_{R} Y_{U}-X_{U} Y_{R}\right), \\
& \Delta_{i Z 2}=\left|\begin{array}{ll}
-2 X_{L} & -2 Y_{L} \\
-2 X_{U} & -2 Y_{U}
\end{array}\right|=4\left(X_{L} Y_{U}-X_{U} Y_{L}\right) \\
& \Delta_{i Z 3}=\left|\begin{array}{ll}
-2 X_{L} & -2 Y_{L} \\
-2 X_{R} & -2 Y_{R}
\end{array}\right|=4\left(X_{L} Y_{R}-X_{R} Y_{L}\right)
\end{aligned}
$$

The general determinant of the first system of equations is the following:

$\Delta_{i}=\left|\begin{array}{lll}-2 X_{L} & -2 Y_{L} & -2 Z_{L} \\ -2 X_{R} & -2 Y_{R} & -2 Z_{R} \\ -2 X_{U} & -2 Y_{U} & -2 Z_{U}\end{array}\right|=-8 \cdot\left(X_{L} Y_{R} Z_{U}+X_{U} Y_{L} Z_{R}+X_{R} Y_{U} Z_{L}-X_{L} Y_{U} Z_{R}-X_{R} Y_{L} Z_{U}-X_{U} Y_{R} Z_{L}\right)$

The solutions of the second $(i=2)$, third $(i=3)$, and fourth $(i=4)$ systems of equations are obtained from the solution of the first system by successive replacement of the notation (Table 1):

Table 1. Replacement of the notation.

\begin{tabular}{ccccccc}
\hline \multicolumn{2}{c}{$i=\mathbf{1}$} & \multicolumn{2}{c}{$i=\mathbf{2}$} & \multicolumn{2}{c}{$i=3$} & $i=4$ \\
\hline $\mathrm{L}$ & $\rightarrow$ & $\mathrm{R}$ & $\rightarrow$ & $\mathrm{U}$ & $\rightarrow$ & $\mathrm{D}$ \\
$\mathrm{R}$ & $\rightarrow$ & $\mathrm{U}$ & $\rightarrow$ & $\mathrm{D}$ & $\rightarrow$ & $\mathrm{L}$ \\
$\mathrm{U}$ & $\rightarrow$ & $\mathrm{D}$ & $\rightarrow$ & $\mathrm{L}$ & $\rightarrow$ & $\mathrm{R}$ \\
$\mathrm{D}$ & $\rightarrow$ & $\mathrm{L}$ & $\rightarrow$ & $\mathrm{R}$ & $\rightarrow$ & $\mathrm{U}$ \\
$\mathrm{E}$ & $\rightarrow$ & $\mathrm{F}$ & $\rightarrow$ & $\mathrm{G}$ & $\rightarrow$ & $\mathrm{H}$ \\
$\mathrm{F}$ & $\rightarrow$ & $\mathrm{G}$ & $\rightarrow$ & $\mathrm{H}$ & $\rightarrow$ & $\mathrm{E}$ \\
$\mathrm{G}$ & $\rightarrow$ & $\mathrm{H}$ & $\rightarrow$ & $\mathrm{E}$ & $\rightarrow$ & $\mathrm{F}$ \\
\hline
\end{tabular}

Substitution of Equations (10)-(12) into Equation (6) defines a quadratic equation with respect to the variable $K: a_{i} K^{2}+b_{i} K+c_{i}=0$, where $i$ corresponds to the choice of a pair of hyperboloids, $i=1,2,3,4$.

$$
a_{i}=M_{i X}^{2}+M_{i Y}^{2}+M_{i Z}^{2}-1, b_{i}=2\left(M_{i X} N_{i X}+M_{i Y} N_{i Y}+M_{i Z} N_{i Z}\right), c_{i}=N_{i X}^{2}+N_{i Y}^{2}+N_{i Z}^{2}
$$

The roots of the quadratic equation are the following $\left(b_{i}{ }^{2}-4 a_{i} c_{i} \geq 0\right)$ :

$$
K_{i 1}=\frac{-b_{i}+\sqrt{b_{i}^{2}-4 a_{i} c_{i}}}{2 a_{i}}, K_{i 2}=\frac{-b_{i}-\sqrt{b_{i}^{2}-4 a_{i} c_{i}}}{2 a_{i}}
$$

In the case that one of the two roots $K_{i 1}$ and $K_{i 2}$ is negative for the same value of $i$, it can be immediately excluded from the solution, and then another root of the equation remains in the algorithm. However, a situation is possible when both roots $K_{i 1}$ and $K_{i 2}$ are positive. In this case, it becomes 
impossible to determine the coordinates of the object. It is for such a fairly common case that the fifth BS has to be used in the 3D positioning system based on the analytical method.

Substitution of roots $K_{i 1}$ and $K_{i 2}$ in Equations (10)-(12) defines eight possible sets of $x ; y ; z$ coordinates of an object, defining eight points in space:

$$
\begin{gathered}
{\left[x_{i 1}=X_{i 1}+x_{C}, y_{i 1}=Y_{i 1}+y_{C}, z_{i 1}=Z_{i 1}+z_{C}\right],} \\
{\left[x_{i 2}=X_{i 2}+x_{C}, y_{i 2}=Y_{i 2}+y_{C}, z_{i 2}=Z_{i 2}+z_{C}\right], \text { where }} \\
X_{i 1}=M_{i X} \cdot K_{i 1}+N_{i X}, Y_{i 1}=M_{i Y} \cdot K_{i 1}+N_{i Y}, Z_{i 1}=M_{i Z} \cdot K_{i 1}+N_{i Z} \\
X_{i 2}=M_{i X} \cdot K_{i 2}+N_{i X}, Y_{i 2}=M_{i Y} \cdot K_{i 2}+N_{i Y}, Z_{i 2}=M_{i Z} \cdot K_{i 2}+N_{i Z}
\end{gathered}
$$

In the absence of TDoA measurement errors from eight calculated sets of $x ; y ; z$-coordinates, the coordinates of four points will be the same. It is this decision that is the true decision. To identify it, we carry out the following steps of the Algorithm 1:

Algorithm 1. Identifying of the true solution

1. Calculation of $48=(8$ points $\times 6$ combinations $)$ distances $D$ between points-candidates for the true value, determined by Equation (16):

$$
\begin{aligned}
& D_{i 1 \_j 1}=\sqrt{\left(x_{i 1}-x_{j 1}\right)^{2}+\left(y_{i 1}-y_{j 1}\right)^{2}+\left(z_{i 1}-z_{j 1}\right)^{2}}, \\
& D_{i 2 \_j 2}=\sqrt{\left(x_{i 2}-x_{j 2}\right)^{2}+\left(y_{i 2}-y_{j 2}\right)^{2}+\left(z_{i 2}-z_{j 2}\right)^{2}} \\
& \text { where both indices } i \text { and } j \text { take the values } 1,2,3,4 \text {, and } i \neq j .
\end{aligned}
$$

2. Sorting the elements of a string consisting of six distances related to one candidate point in ascending order.

3. Summing the first three elements of the row.

4. For all eight candidate points, writing the sum of the first three elements to a line. The meaning of each sum is the sum of the distances between the three nearest points.

5. In parallel with step 4 of the algorithm, forming three lines by $x ; y ; z$ from eight elements in each line. Each of the eight line elements corresponds to the coordinates of the candidate points.

6. Making a cycle on 5 configurations of the spatial arrangement of BS. That is, each BS once becomes a reference. For each configuration, its own line of eight "sums of minimum distances" is formed. The strings are combined into a matrix.

7. In the resulting matrix of "sums of minimum distances", seeking the element with the minimum value and its indices in the matrix.

8. In parallel with item 7, forming three coordinate matrices from the rows of item 5. Each row of the matrix corresponds to the configuration of the location of the BS.

9. Selecting, from the matrix of item 8 by the indices of item 7 , the coordinates of the estimation of the position of the object $\bar{x} ; \bar{y} ; \bar{z}$. The selected coordinates are the closest to the true coordinates of the target. The rest of the coordinates are either false or determine the coordinates of the target with a greater error.

\section{Results}

\subsection{Spatial Ambiguity Problem}

In [67], an example of calculating target coordinates is given, in which the values of $K_{i 1}$ and $K_{i 2}$ are both positive. Two variants of target coordinates were obtained. The authors chose one of the options based on the disposition of the system of receivers to determine final target coordinates; however, they did not specify what kind of disposition it is. Most likely, it meant the negative $z$ coordinate of the target, which means height. It should be noted that $K_{i 1}$ and $K_{i 2}$ are both positive and often occur with a positive $z$ coordinate. In this case, the algorithm [56] is generally unable to give the correct values of the target coordinates. The method of linearizing hyperbolic equations also leads to a gross error in determining the coordinates of the object. In this article, a method is proposed that eliminates the ambiguity of determining the coordinates.

Figure 2 shows the areas in which $K_{i 1}$ and $K_{i 2}$ are both positive, and therefore, there is no way to determine the true value of the target coordinates. The calculations were carried out at BS coordinates $(-40 ; 40 ; 0),(40 ; 40 ; 10),(40 ;-40 ; 20),(-40 ;-40 ; 30) \mathrm{m}$. In Figure 2 , sensor projections on the horizontal plane are marked with blue dots. The target height took the values $0 \mathrm{~m}(\mathrm{a}) ; 15 \mathrm{~m} \mathrm{(b);30} \mathrm{m}(\mathrm{s})$. If the 
heights of the sensors differ slightly, the undetectable areas expand significantly $\left(z_{C, L, R, U}=8 ; 10 ; 12\right.$; $14 \mathrm{~m}$, Figure $2 \mathrm{~d}$ ). With the same BS heights, the target positions are not determined anywhere using the algorithm [67]. Significant areas, where the target coordinates are not determined, exist for almost all values of the BS coordinates.

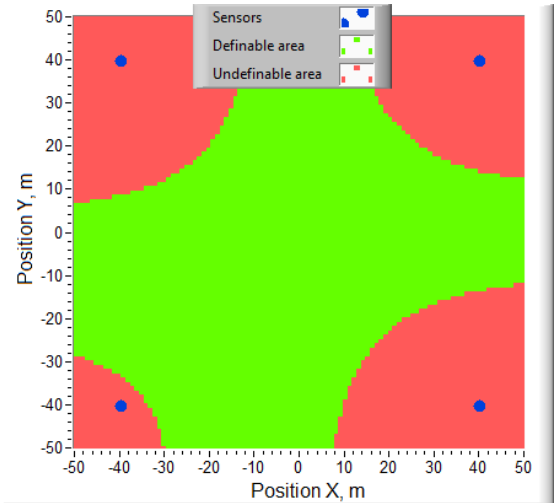

(a)

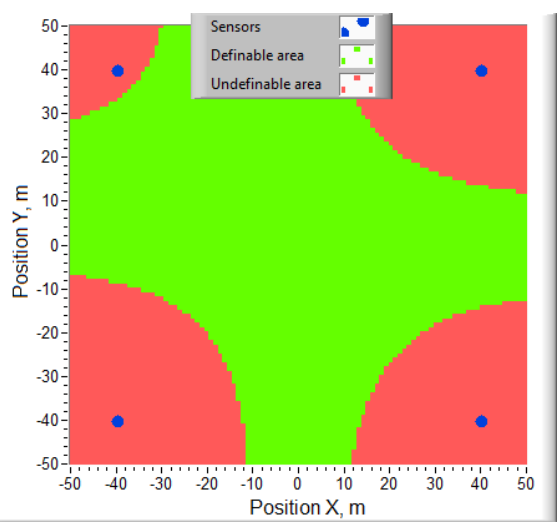

(c)

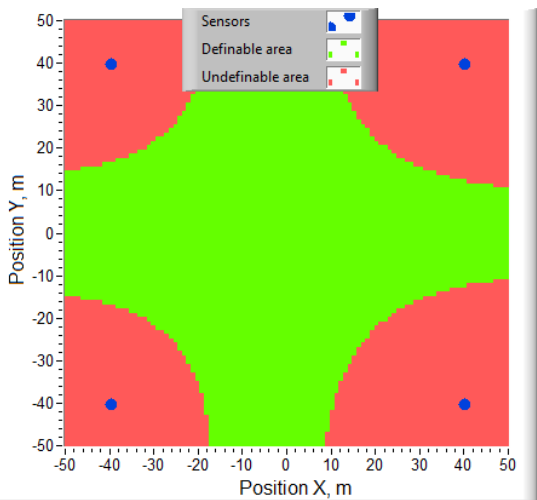

(b)

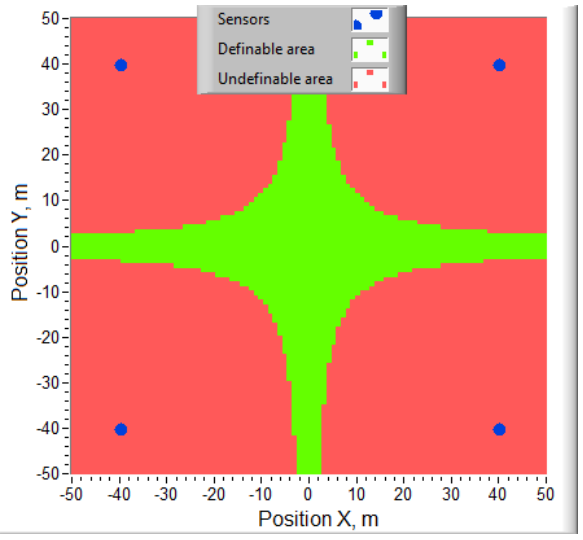

(d)

Figure 2. Areas in which the target coordinates are not determined by the algorithm [67]: (a) $\mathrm{z}=0 \mathrm{~m}$; (b) $\mathrm{z}=15 \mathrm{~m}$; (c) $\mathrm{z}=30 \mathrm{~m}$; (d) $\mathrm{z}=0 \mathrm{~m}$ when $\mathrm{z}_{C, L, R, U}=8 ; 10 ; 12 ; 14 \mathrm{~m}$.

The method proposed by us is free from the indicated problem of ambiguity in determining the coordinates of an object. The method allows you to determine the coordinates of the object at all points in Figure 2.

\subsection{Influence of the TDoA Fluctuations on the Accuracy of Coordinate Estimation}

TDoA measurement errors occur mainly due to time desynchronization at base stations, as well as the conditions of propagation and reception of radio signals, and are the main reason for inaccurate determination of the object's position. Using a computer experiment in the LabVIEW environment, we investigated the dependence of the root mean square (RMS) deviation of the object coordinates on the standard deviation of the TDoA of microwave signals in the positioning radar. For this, Gaussian white noise with the same standard deviation value for all BSs was added to the TDoA value at the input of each receiver of the positioning system. To determine the standard deviation of the object position estimate, $n=500$ computational experiments were carried out for each value of the standard deviation of the Gaussian white noise. The standard deviation of the object coordinates estimate was determined in accordance with the expression:

$$
R M S=\sqrt{\frac{1}{n} \sum_{k=1}^{n}\left[\left(\bar{x}_{k}-x\right)^{2}+\left(\bar{y}_{k}-y\right)^{2}+\left(\bar{z}_{k}-z\right)^{2}\right]}
$$


where index $k$-experiment number, $\left[\bar{x}_{k} ; \bar{y}_{k} ; \bar{z}_{k}\right]$ —estimation of object coordinates in $k$ experiment, $[x ; y ; z]$-object's true coordinates.

Figure 3 (time is in picoseconds) shows the dependences of the RMS deviation of the object coordinates estimate on the standard deviation of the Gaussian white noise of the difference in the arrival times of microwave signals to the BS for the matrix linearization algorithm and the proposed analytical algorithm. Dependents were calculated for object coordinates $[x ; y ; z]=[10 ; 1 ; 5]$ and coordinates of $\mathrm{BS}_{C}\left[x_{C} ; y_{C} ; z_{C}\right]=[-40 ; 40 ; 0], \mathrm{BS}_{L}\left[x_{L} ; y_{L} ; z_{L}\right]=[40 ; 40 ; 5], \mathrm{BS}_{R}\left[x_{R} ; y_{R} ; z_{R}\right]=[40 ;-40 ; 10]$, $\mathrm{BS}_{U}\left[x_{U} ; y_{U} ; z_{U}\right]=[-40 ;-40 ; 15]$ and $\mathrm{BS}_{D}\left[x_{D} ; y_{D} ; z_{D}\right]=[40 ; 40 ; 20] \mathrm{m}$.

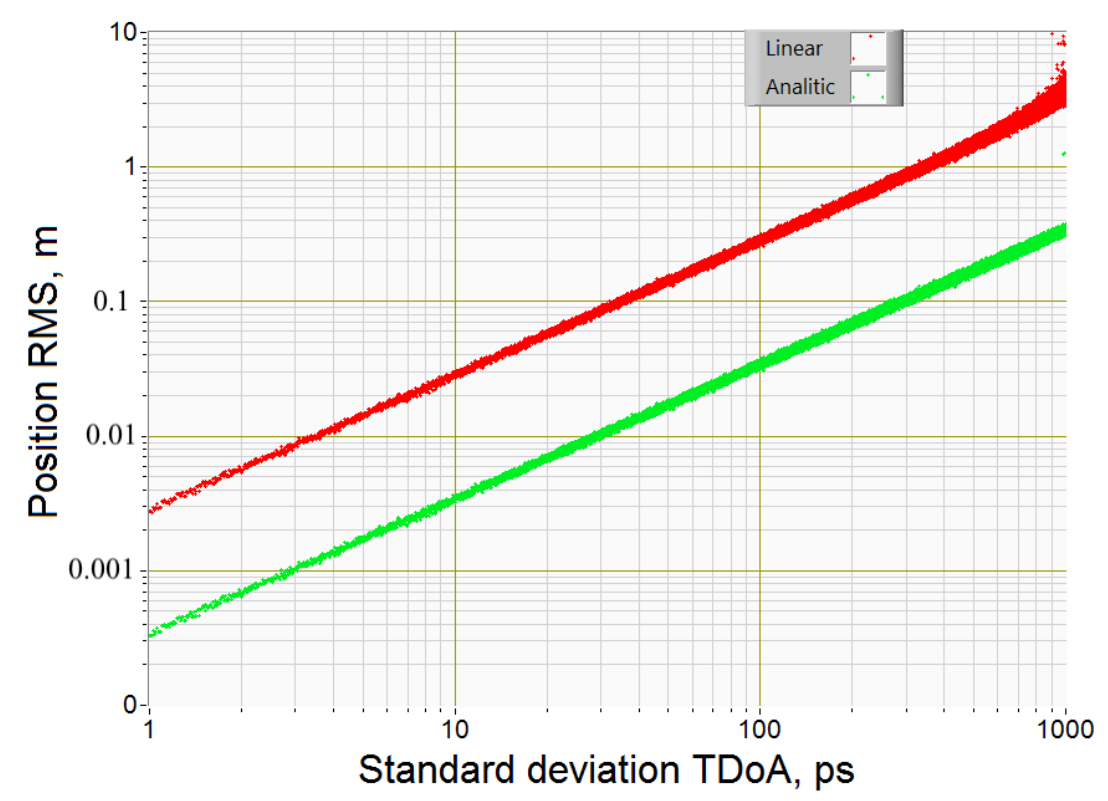

Figure 3. Dependence of the mean square deviation of the object coordinates estimate on the standard deviation of the Gaussian white noise of the difference in arrival time ( 1 picosecond $\left.(\mathrm{ps})=10^{-12} \mathrm{~s}\right)$ : red points for the matrix linearization algorithm, green points for the analytical algorithm proposed.

From the simulation results, it follows that the proposed algorithm provides the accuracy of determining the coordinates of the object 10 times higher than the method of linearization of hyperbolic equations. The proposed method works with high accuracy $(5 \mathrm{~cm})$ with BS standard deviation of TDoA up to $200 \mathrm{ps}$, while the linearization method with such BS standard deviation of TDoA provides an accuracy of $60 \mathrm{~cm}$. Gross failures in the linearization algorithm start at 500 ps standard deviation of TDoA, while the proposed analytical algorithm works without gross failures at $1000 \mathrm{ps}$ standard deviation of TDoA.

The following RMS dependences of the coordinate estimates were obtained for the configuration of five BSs located on a circle as in [74] with a radius of $50 \mathrm{~m}$, which corresponds to the Local Positioning System (LPS). Coordinates were as follows: $\mathrm{BS}_{C}\left[x_{C} ; y_{C} ; z_{C}\right]=[29.4 ;-40.45 ; 20], \mathrm{BS}_{L}\left[x_{L} ; y_{L} ; z_{L}\right]=$ $[-29.4 ;-40.45 ; 25], \mathrm{BS}_{R}\left[x_{R} ; y_{R} ; z_{R}\right]=[-47.55 ; 15.45 ; 30], \mathrm{BS}_{U}\left[x_{U} ; y_{U} ; z_{U}\right]=[0 ; 50 ; 0]$, and $\mathrm{BS}_{D}\left[x_{D} ; y_{D} ;\right.$ $\left.z_{D}\right]=[47.55 ; 15.45 ; 10] \mathrm{m}$, which is presented in Figure 4. Target height is $\mathrm{z}=5 \mathrm{~m}$. The number of computational experiments $n=100$. 


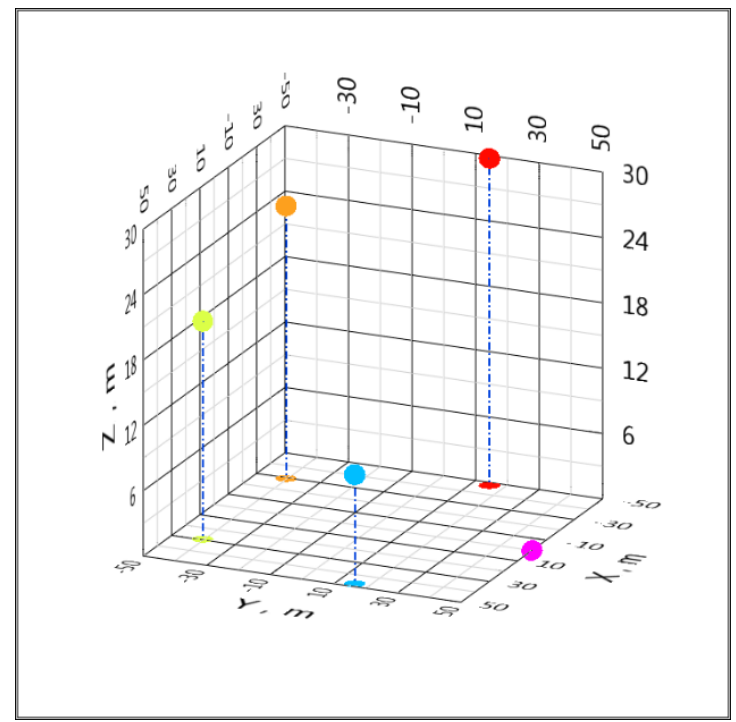

Figure 4. The location of the BS in the example of a local positioning system (LPS) system.

It should be noted that the algorithm with one equation [56], as well as the linearization method, produces a gross error at the points of the 3D space at which the determinant of the system of equations becomes zero. An example of such a situation is shown in Figure 5.

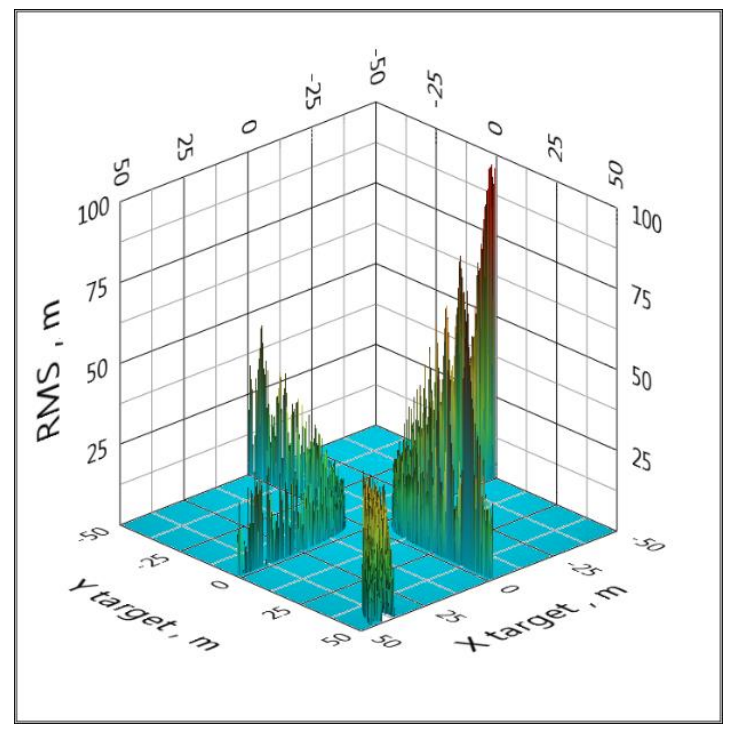

Figure 5. An example of gross errors at the points at which the determinant of the system of equations vanishes (LPS).

An effective way to eliminate such catastrophic errors is to use a cycle of five configurations of the spatial arrangement of BS, that is, to replace the reference BS. If a configuration with some reference BS has the effect shown in Figure 5, then when changing the reference BS, this effect will no longer be present. This method is implemented in clause 6 of the proposed algorithm. All graphs below are calculated using a cycle for five configurations of the spatial arrangement of the BS (Figures 6 and 7). 


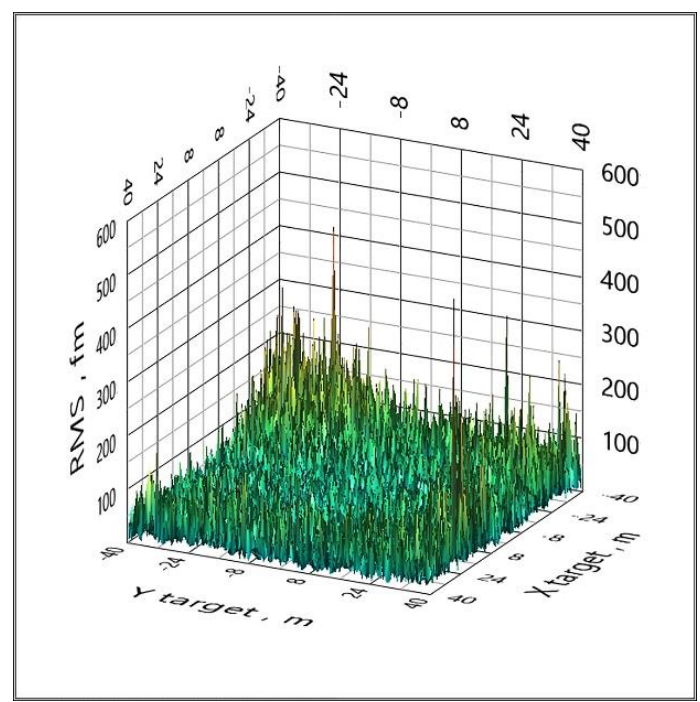

Figure 6. The distribution of the RMS ( 1 femtometer $\left.(\mathrm{fm})=10^{-15} \mathrm{~m}\right)$ coordinate estimate over the area when Gaussian noise is added to the TDoA values with a standard deviation of 0 ps (LPS).

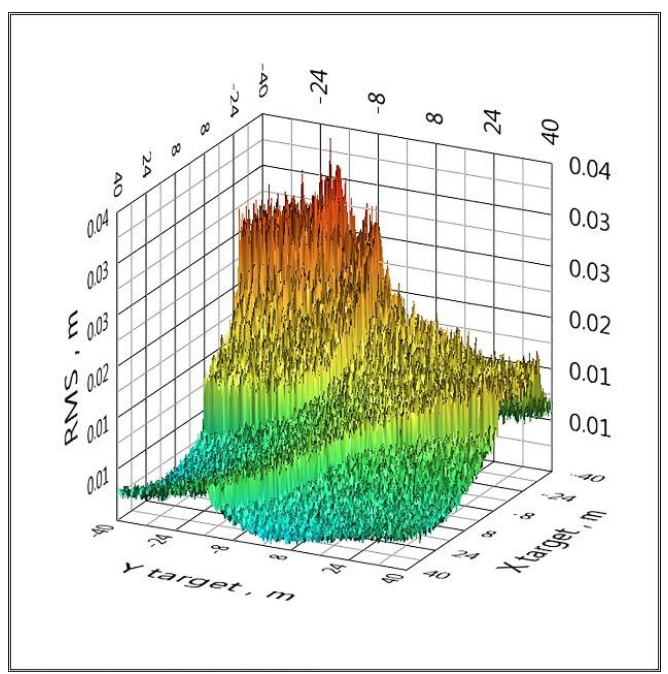

Figure 7. The distribution of the RMS coordinate estimate over the area when Gaussian noise is added to the TDoA values with a standard deviation of $10 \mathrm{ps}$ (LPS).

The RMS area distributions when Gaussian noise is added to the TDoA values with a standard deviation of more than 10 ps have a similar form to Figure 7, and the differences lie in the values of $\mathrm{RMS}_{\min }$ and $\mathrm{RMS}_{\max }$, which are summarized in Table 2. $\mathrm{RMS}_{\min }$ for the linearization method is approximately three times higher than for the analytical method; however, there are gross errors associated with zeroing the determinant of the system at some points in space. Therefore, $\mathrm{RMS}_{\min }$ and $\mathrm{RMS}_{\max }$ for the linearization method are not included in Table 1.

Table 2. $\mathrm{RMS}_{\min }$ and $\mathrm{RMS}_{\max }$ for analytical method (LPS).

\begin{tabular}{ccc}
\hline Deviation TDoA, ps & RMSmin, $\mathbf{m}$ & RMSmax, $\mathbf{m}$ \\
\hline 0 & 0 & $5.56 \times 10^{-13}$ \\
10 & 0.0032 & 0.037 \\
100 & 0.031 & 0.338 \\
1000 & 0.328 & 3.3 \\
\hline
\end{tabular}


As can be seen from the graphs, the accuracy of determining the coordinates strongly depends on the choice of the BS position.

Configurations of global satellite positioning systems are of practical interest. Let us apply the proposed algorithm to simulate the accuracy of determining coordinates in the global positioning system (GPS). Orbiting satellites of the system should be perceived as BS, and the object, the coordinates of which are determined, is on the Earth. We will accept the coordinates of the BS (satellites) as follows: $\mathrm{BS}_{C}\left[x_{C} ; y_{C} ; z_{C}\right]=\left[0 ; 1.53 \times 10^{7} ; 1.98 \times 10^{7}\right], \mathrm{BS}_{L}\left[x_{L} ; y_{L} ; z_{L}\right]=\left[1.45 \times 10^{7} ; 4.71 \times 10^{6} ; 2 \times 10^{7}\right]$, $\mathrm{BS}_{R}\left[x_{R} ; y_{R} ; z_{R}\right]=\left[8.97 \times 10^{6} ;-1.23 \times 10^{7} ; 2.02 \times 10^{7}\right], \mathrm{BS}_{U}\left[x_{U} ; y_{U} ; z_{U}\right]=\left[-8.97 \times 10^{6} ;-1.23 \times 10^{7} ;\right.$ $\left.2.04 \times 10^{7}\right]$ and $\mathrm{BS}_{D}\left[x_{D} ; y_{D} ; z_{D}\right]=\left[-1.45 \times 10^{7} ; 4.71 \times 10^{6} ; 2.06 \times 10^{7}\right] \mathrm{m}$, which is presented in Figure 8. The target height is $\mathrm{z}=0 \mathrm{~m}$. The number of computational experiments $\mathrm{n}=100$. The satellites are equipped with atomic clocks with a daily instability of no worse than $10^{-13}$; therefore, in the calculations, we limited ourselves to the maximum value of the standard deviation of the Gaussian noise TDoA 10 ps (Figures 9 and 10). Such a model can be viewed as a simplified model of the location of satellites and targets on Earth in a GPS positioning system.

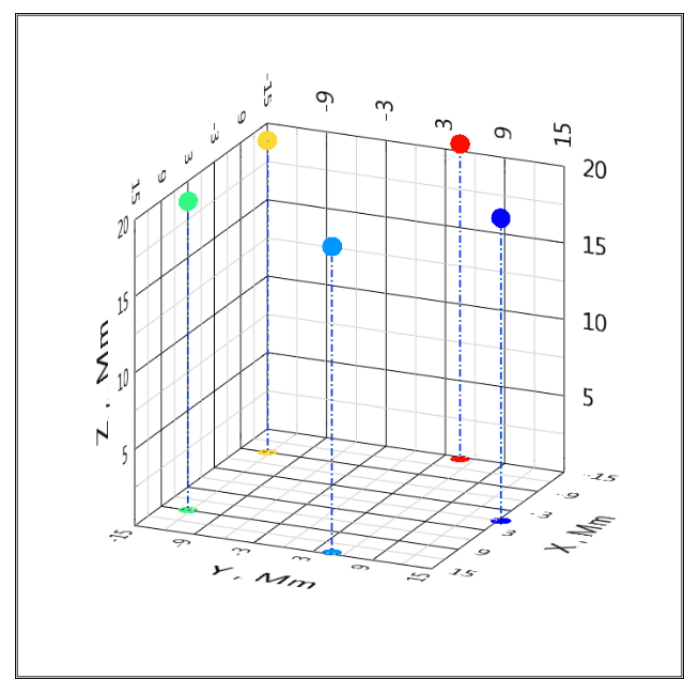

Figure 8. Simplified model of the location $\left(1\right.$ megameter $\left.(\mathrm{Mm})=10^{6} \mathrm{~m}\right)$ of satellites (red) and a target on Earth (blue) in a GPS positioning system.

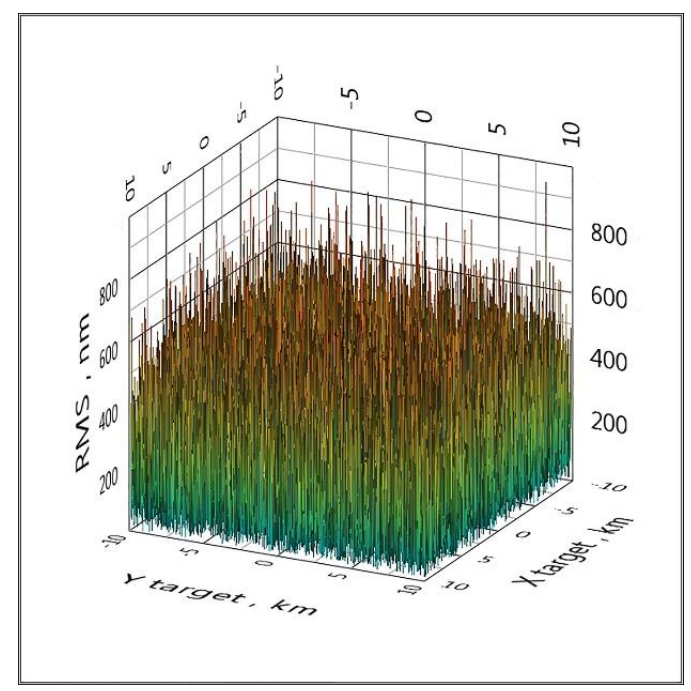

Figure 9. The distribution of the RMS coordinate $\left(1\right.$ nanometer $\left.(\mathrm{nm})=10^{-9} \mathrm{~m}\right)$ estimate over the area when Gaussian noise is added to the TDoA values with a standard deviation of 0 ps (GPS). 


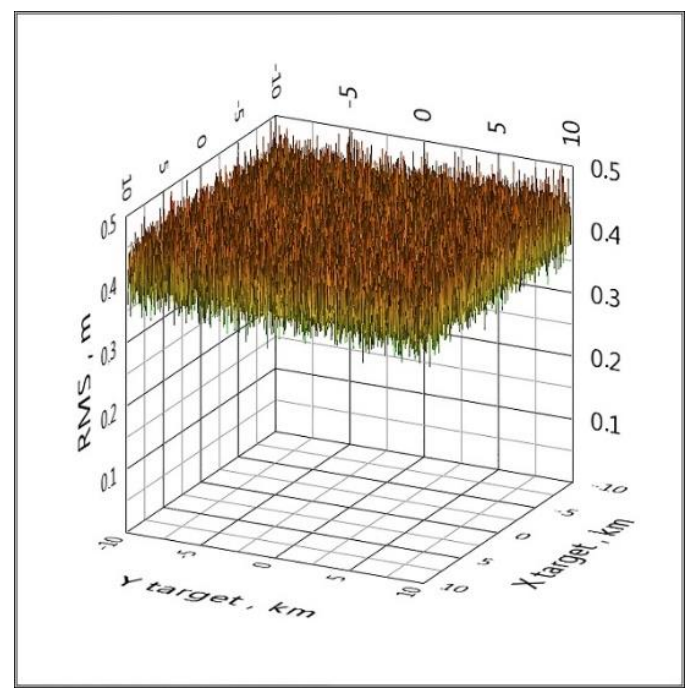

Figure 10. The distribution of the RMS coordinate estimate over the area by adding Gaussian noise to the TDoA values with a standard deviation of $10 \mathrm{ps}$ (GPS).

$\mathrm{RMS}_{\min }$ and $\mathrm{RMS}_{\max }$ for the analytical method and linearization method for the last problem (GPS) are summarized in Table 3.

Table 3. $\mathrm{RMS}_{\min }$ and $\mathrm{RMS} \mathrm{S}_{\max }$ for analytical and linear methods (GPS).

\begin{tabular}{ccccc}
\hline Deviation TDoA, ps & $\begin{array}{c}\text { RMSmin, m. } \\
\text { Analytical Method }\end{array}$ & $\begin{array}{c}\text { RMSmin, m. } \\
\text { Linear Method }\end{array}$ & $\begin{array}{c}\text { RMSmax, m. } \\
\text { Analytical Method }\end{array}$ & $\begin{array}{c}\text { RMSmax, m. } \\
\text { Linear Method }\end{array}$ \\
\hline 0 & 0 & $1.91 \times 10^{-9}$ & $1.013 \times 10^{-6}$ & $8.80 \times 10^{-5}$ \\
1 & 0.029 & 0.166 & 0.055 & 0.318 \\
10 & 0.29 & 1.67 & 0.552 & 3.17 \\
\hline
\end{tabular}

It can be seen that the proposed algorithm is significantly superior to the widely used positioning algorithm based on the linearization of hyperbolic equations.

\section{Discussion}

The new method, based on the analytical solution of hyperbolic equations, in contrast to many existing methods, makes it possible to obtain an accurate solution for determining the coordinates of an object based on the TDoA measurement. Considerable efforts of researchers have been aimed at finding ways to transform expressions with radicals into linear relations and to ensure fast convergence of solutions. In part, this direction of research is explained by the limited capabilities of the processor technology and the complexity of the implementation of computations of expressions with radicals, which is necessary in the analytical exact method that we have proposed. Of course, the limited mathematical capabilities of inexpensive processors is the main limitation to the widespread implementation of the proposed method. However, the computational element base, both based on processors and FPGAs, is rapidly developing, and we should expect in the near future the appearance of inexpensive processors that allow calculating mathematical functions with radicals in real-time. In addition, in some positioning systems, the function of mathematical processing can be transferred to a system controller (server), in the software of which the proposed method is implemented. In these cases, the restriction on the possibilities of mathematical data processing is removed.

To accurately determine the moment of arrival of a radio signal from an object to base stations, various forms of radio signals are used. The wider the spectrum of the radio signal, the more accurately it is localized in time. Therefore, ultra-wideband (UWB) signals with a wide spectrum have advantages over signals with a shorter spectrum and are used in positioning systems [75]. In our article, the question 
of the form of the used radio signal is not a subject of research. Radio signal propagation conditions impose a significant influence on the functioning of positioning systems. Multipath propagation, broadband interference, and atmospheric phenomena in global positioning systems lead to TDoA fluctuations. In our work, we did not consider the issues of propagation and reception of radio signals. In our model, the standard deviation of the TDoA is used as a measure of fluctuations, the value of which is determined by the factors listed above.

Within the framework of the adopted model, we have established that the proposed method has the ability to exclude spatial zones of ambiguity in determining the coordinates, which are characteristic of approximate analytical algorithms with one system of equations. The algorithm also eliminates the gross error at the points of the 3D space, at which the determinant of the system of equations becomes zero.

In future work, we propose to calculate the Cramer-Rao Lower Bound (CRLB) for the proposed method, carry out practice implementations to compare the performance with the simulations results, investigate the resistance of the method to non-line-of-sight (NLOS) radio signal propagation, and also consider the applicability of the method for determining the coordinates of a set of objects.

\section{Conclusions}

Modern systems of local and global positioning put forward high requirements for the accuracy of determining the coordinates of objects. This article proposes an analytical method for the exact solution of a system of hyperbolic equations, which, with a high accuracy of less than a few $\mathrm{cm}$, allows one to determine the coordinates of objects and at the same time exclude spatial zones in which approximate analytical algorithms with one system of equations are unable to unambiguously determine the coordinates of an object or give a gross error at the points of the 3D space, at which point the determinant of the system of equations becomes zero. The absence of any omitted terms in the solution provided the developed algorithm with significant resistance to fluctuations in TDoA caused by the conditions of propagation and reception of radio signals and time desynchronization of the BS while maintaining a high accuracy in determining the coordinates. It was found that the proposed method works with high accuracy $(5 \mathrm{~cm})$ with BS standard deviation of TDoA in time up to 200 ps, while the linearization method with such BS standard deviation of TDoA provides an accuracy of $60 \mathrm{~cm}$ for LPS. The performance of the algorithm has been confirmed by computational experiments for both LPS and GPS.

Author Contributions: Formal analysis, S.I.; Investigation, V.B.; Methodology, V.K.; Software, A.F. V.K. conceived the proposed method. All authors discussed and designed the proposed algorithm. All authors have read and agreed to the published version of the manuscript.

Funding: The reported study was funded by RFBR, project number 19-29-06034.

Conflicts of Interest: The authors declare no conflict of interest.

\section{References}

1. Weon, I.S.; Lee, S.G.; Ryu, J.K. Object Recognition Based Interpolation with 3D LIDAR and Vision for Autonomous Driving of an Intelligent Vehicle. IEEE Access 2020, 8, 65599-65608. [CrossRef]

2. López-Sastre, R.J;; Herranz-Perdiguero, C.; Guerrero-Gómez-Olmedo, R.; Oñoro-Rubio, D.; Maldonado-Bascón, S. Boosting multi-vehicle tracking with a joint object detection and viewpoint estimation sensor. Sensors 2019, 19, 4062. [CrossRef]

3. Wang, G.; So, A.M.C.; Li, Y. Robust convex approximation methods for TDOA-based localization under NLOS conditions. IEEE Trans. Signal Process. 2016, 64, 3281-3296. [CrossRef]

4. Gan, Y.; Cong, X.; Sun, Y. Refinement of TOA localization with sensor position uncertainty in closed-form. Sensors 2020, 20, 390. [CrossRef] [PubMed]

5. Nguyen, N.H.; Doğançay, K. Optimal geometry analysis for multistatic TOA localization. IEEE Trans. Signal Process. 2016, 64, 4180-4193. [CrossRef] 
6. Deak, G.; Curran, K.; Condell, J. A survey of active and passive indoor localisation systems. Comput. Commun. 2012, 35, 1939-1954. [CrossRef]

7. Ferreira, A.F.G.; Fernandes, D.M.A.; Catarino, A.P.; Monteiro, J.L. Localization and positioning systems for emergency responders: A survey. IEEE Commun. Surv. Tutor. 2017, 19, 2836-2870. [CrossRef]

8. Hashemi, A.; Cao, Y.; Casbeer, D.W.; Yin, G. Unmanned aerial vehicle circumnavigation using noisy range-based measurements without global positioning system information. J. Dyn. Syst. Meas. Control 2015, 137, 031009. [CrossRef]

9. Gharghan, S.K.; Nordin, R.; Ismail, M.; Abd Ali, J. Accurate wireless sensor localization technique based on hybrid PSO-ANN algorithm for indoor and outdoor track cycling. IEEE Sens. J. 2015, 16, 529-541. [CrossRef]

10. Sadowski, S.; Spachos, P. Rssi-based indoor localization with the internet of things. IEEE Access 2018, 6, 30149-30161. [CrossRef]

11. Ramadhan, H.; Yustiawan, Y.; Kwon, J. Applying Movement Constraints to BLE RSSI-Based Indoor Positioning for Extracting Valid Semantic Trajectories. Sensors 2020, 20, 527. [CrossRef] [PubMed]

12. Nguyen, N.H.; Doğançay, K. Instrumental variable based Kalman filter algorithm for three-dimensional AOA target tracking. IEEE Signal Process. Lett. 2018, 25, 1605-1609. [CrossRef]

13. Mallick, M.; Arulampalam, S.; Yan, Y.; Ru, J. Three-Dimensional Tracking of an Aircraft Using Two-Dimensional Radars. IEEE Trans. Aerosp. Electron. Syst. 2018, 54, 585-600. [CrossRef]

14. Talebi, S.P.; Werner, S. Distributed Kalman filtering and control through embedded average consensus information fusion. IEEE Trans. Autom. Control 2019, 64, 4396-4403. [CrossRef]

15. Luo, J.A.; Pan, S.W.; Peng, D.L.; Wang, Z.; Li, Y.J. Source localization in acoustic sensor networks via constrained least-squares optimization using AOA and GROA measurements. Sensors 2018, 18, 937. [CrossRef]

16. Kazmi, W.; Foix, S.; Alenyà, G.; Andersen, H.J. Indoor and outdoor depth imaging of leaves with time-of-flight and stereo vision sensors: Analysis and comparison. ISPRS J. Photogramm. Remote Sens. 2014, 88, 128-146. [CrossRef]

17. Iliev, N.; Paprotny, I. Review and comparison of spatial localization methods for low-power wireless sensor networks. IEEE Sens. J. 2015, 15, 5971-5987. [CrossRef]

18. Francis, S.L.; Anavatti, S.G.; Garratt, M. Real-time path planning module for autonomous vehicles in cluttered environment using a 3D camera. Int. J. Veh. Auton. Syst. 2018, 14, 40-61. [CrossRef]

19. Surti, S.; Karp, J.S. Advances in time-of-flight PET. Phys. Medica 2016, 32, 12-22. [CrossRef]

20. Paredes, J.A.; Álvarez, F.J.; Aguilera, T.; Aranda, F.J. Precise drone location and tracking by adaptive matched filtering from a top-view ToF camera. Expert Syst. Appl. 2020, 141, 112989. [CrossRef]

21. Wang, Y.; Ma, S.; Chen, C.P. TOA-based passive localization in quasi-synchronous networks. IEEE Commun. Lett. 2014, 18, 592-595. [CrossRef]

22. Liu, Y.; Guo, F.; Yang, L.; Jiang, W. Source localization using a moving receiver and noisy TOA measurements. Signal Process. 2016, 119, 185-189. [CrossRef]

23. Xiong, H.; Peng, M.; Gong, S.; Du, Z. A novel hybrid RSS and TOA positioning algorithm for multi-objective cooperative wireless sensor networks. IEEE Sens. J. 2018, 18, 9343-9351. [CrossRef]

24. Wei, Y.; Li, W.; Tang, Q.; Wei, P.; Zhang, H. A Closed-Form Location Algorithm Without Auxiliary Variables for Moving Target in Noncoherent Multiple-Input and Multiple-Output Radar System. IEEE Access 2020, 8 , 69496-69508. [CrossRef]

25. Wang, Y.; Ho, K.C. Unified near-field and far-field localization for AOA and hybrid AOA-TDOA positionings. IEEE Ttans. Wirel. Commun. 2017, 17, 1242-1254. [CrossRef]

26. Zhang, W.; Zhang, G. Geolocation Algorithm of Interference Sources from FDOA Measurements Using Satellites Based on Taylor Series Expansion. In Proceedings of the IEEE Vehicular Technology Conference, Nanjing, China, 15-18 May 2016.

27. Zhang, S.; Huang, Z.; Feng, X.; He, J.; Shi, L. Multi-Sensor Passive Localization Using Second Difference of Coherent Time Delays with Incomplete Measurements. IEEE Access 2019, 7, 43167-43178. [CrossRef]

28. Zou, Y.; Liu, H.; Wan, Q. An iterative method for moving target localization using TDOA and FDOA measurements. IEEE Access 2017, 6, 2746-2754. [CrossRef]

29. Hmam, H. Optimal sensor velocity configuration for TDOA-FDOA geolocation. IEEE Trans. Signal Process. 2016, 65, 628-637. [CrossRef]

30. Wang, D.; Zhang, P.; Yang, Z.; Wei, F.; Wang, C. A novel estimator for TDOA and FDOA positioning of multiple disjoint sources in the presence of calibration emitters. IEEE Access 2019, 8, 1613-1643. [CrossRef] 
31. Kim, S.; Chong, J.W. An efficient TDOA-based localization algorithm without synchronization between base stations. Int. J. Distrib. Sens. Netw. 2015, 11, 832351. [CrossRef]

32. Wang, D.; Yin, J.; Tang, T.; Chen, X.; Wu, Z. Quadratic constrained weighted least-squares method for TDOA source localization in the presence of clock synchronization bias: Analysis and solution. Digit. Signal Process. 2018, 82, 237-257. [CrossRef]

33. Zheng, Z.; Zhang, H.; Wang, W.Q.; So, H.C. Source localization using TDOA and FDOA measurements based on semidefinite programming and reformulation linearization. J. Frankl. Inst. 2019, 356, 11817-11838. [CrossRef]

34. Wang, Y.; Wu, Y. An efficient semidefinite relaxation algorithm for moving source localization using TDOA and FDOA measurements. IEEE Commun. Lett. 2016, 21, 80-83. [CrossRef]

35. Hu, D.; Huang, Z.; Chen, X.; Lu, J. A moving source localization method using TDOA, FDOA and Doppler rate measurements. IEICE Trans. Commun. 2016, 99, 758-766. [CrossRef]

36. Wennervirta, J.; Wigren, T. RTT positioning field performance. IEEE Trans. Veh. Technol. 2010, 59, 3656-3661. [CrossRef]

37. Wigren, T. Fingerprinting localisation using round trip time and timing advance. IET Commun. 2012, 6, 419-427. [CrossRef]

38. Guo, G.; Chen, R.; Ye, F.; Peng, X.; Liu, Z.; Pan, Y. Indoor Smartphone Localization: A Hybrid WiFi RTT-RSS Ranging Approach. IEEE Access 2019, 7, 176767-176781. [CrossRef]

39. Tahat, A.; Kaddoum, G.; Yousefi, S.; Valaee, S.; Gagnon, F. A look at the recent wireless positioning techniques with a focus on algorithms for moving receivers. IEEE Access 2016, 4, 6652-6680. [CrossRef]

40. Gezici, S. A survey on wireless position estimation. Wirel. Pers. Commun. 2008, 44, 263-282. [CrossRef]

41. Stefanski, J.; Sadowski, J. TDOA versus ATDOA for wide area multilateration system. EURASIP J. Wirel. Commun. Netw. 2018, 2018, 179. [CrossRef]

42. Díez-González, J.; Álvarez, R.; González-Bárcena, D.; Sánchez-González, L.; Castejón-Limas, M.; Perez, H. Genetic Algorithm Approach to the 3D Node Localization in TDOA Systems. Sensors 2019, 19, 3880. [CrossRef] [PubMed]

43. Ge, T.; Tharmarasa, R.; Lebel, B.; Florea, M.; Kirubarajan, T.T. A Multidimensional TDOA Association Algorithm for Joint Multitarget Localization and Multisensor Synchronization. IEEE Trans. Aerosp. Electron. Syst. 2019, 56, 2083-2100. [CrossRef]

44. Yaro, A.S.; Sha'ameri, A.Z.; Kamel, N. Position Estimation Error Performance Model for a Minimum Configuration 3-D Multilateration. Int. J. Electr. Eng. Inform. 2018, 10, 153-169. [CrossRef]

45. Mao, G.; Fidan, B.; Anderson, B.D. Wireless sensor network localization techniques. Comput. Netw. 2007, 51, 2529-2553. [CrossRef]

46. Ho, K.C. Bias reduction for an explicit solution of source localization using TDOA. IEEE Trans. Signal Process. 2012, 60, 2101-2114. [CrossRef]

47. Chan, Y.T.; Ho, K.C. A simple and efficient estimator for hyperbolic location. IEEE Trans. Signal Process. 1994, 42, 1905-1915. [CrossRef]

48. Okello, N.; Fletcher, F.; Musicki, D.; Ristic, B. Comparison of recursive algorithms for emitter localisation using TDOA measurements from a pair of UAVs. IEEE Trans. Aerosp. Electron. Syst. 2011, 47, 1723-1732. [CrossRef]

49. Díez-González, J.; Álvarez, R.; Sánchez-González, L.; Fernández-Robles, L.; Pérez, H.; Castejón-Limas, M. 3D Tdoa Problem Solution with Four Receiving Nodes. Sensors 2019, 19, 2892. [CrossRef]

50. Sun, Y.; Ho, K.C.; Wan, Q. Solution and Analysis of TDOA Localization of a Near or Distant Source in Closed Form. IEEE Trans. Signal Process. 2019, 67, 320-335. [CrossRef]

51. Peng, Z.; Li, C. Portable microwave radar systems for short-range localization and life tracking: A review. Sensors 2019, 19, 1136. [CrossRef]

52. Ivanov, S.I.; Kuptsov, V.D.; Fedotov, A.A. The signal processing algorithm of automotive FMCW radars with an extended range of speed estimation. J. Phys. Conf. Ser. 2019, 1236, 012081. [CrossRef]

53. Kuptsov, V.D.; Ivanov, S.I.; Fedotov, A.A.; Badenko, V.L. Features of Multi-target Detection Algorithm for Automotive FMCW Radar. In Lecture Notes in Computer Science; Springer: Berlin/Heidelberg, Germany, 2019; Volume 11660, pp. 355-364.

54. Kronauge, M.; Rohling, H. New chirp sequence radar waveform. IEEE Trans. Aerosp. Electron. Syst. 2014, 50, 2870-2877. [CrossRef]

55. Sun, S.; Petropulu, A.P.; Poor, H.V. MIMO Radar for Advanced Driver-Assistance Systems and Autonomous Driving: Advantages and Challenges. IEEE Signal Process. Mag. 2020, 37, 98-117. [CrossRef] 
56. Ivanov, S.I.; Kuptsov, V.D.; Fedotov, A.A.; Badenko, V.L. CFAR multi-target detection based on non-central Chi-square distribution for FMCW. J. Phys. Conf. Ser. 2020, 1515, 032059. [CrossRef]

57. Kuptsov, V.D.; Ivanov, S.I.; Fedotov, A.A.; Badenko, V.L. Multi-target method for small unmanned vehicles parameters remote determination by microwave radars. J. Phys. Conf. Ser. 2020, 1515, 032045. [CrossRef]

58. Kutsov, V.D.; Ivanov, S.I.; Fedotov, A.A.; Badenko, V.L. Millimeter Wave Radar for Intelligent Transportation Systems: A Case Study of Multi-Target Problem Solution. E3S Web Conf. 2020, 157, 05011. [CrossRef]

59. Guo, X.; Ansari, N.; Hu, F.; Shao, Y.; Elikplim, N.R.; Li, L. A survey on fusion-based indoor positioning. IEEE Commun. Surv. Tutor. 2019, 22, 566-594. [CrossRef]

60. Fang, B.T. Simple solutions for hyperbolic and related position fixes. IEEE Trans. Aerosp. Electron. Syst. 1990, 26, 748-753. [CrossRef]

61. Al-Samahi, S.S.; Zhang, Y.; Ho, K.C. Elliptic and hyperbolic localizations using minimum measurement solutions. Signal Process. 2020, 167, 107273. [CrossRef]

62. Malanowski, M.; Kulpa, K. Two methods for target localization in multistatic passive radar. IEEE Trans. Aerosp. Electron. Syst. 2012, 48, 572-580. [CrossRef]

63. Shuster, S.; Sinclair, A.J.; Lovell, T.A. Initial Relative-Orbit Determination Using Heterogeneous TDOA. In Proceedings of the 2017 IEEE Aerospace Conference, Big Sky, MT, USA, 4-11 March 2017.

64. Cameron, K.J.; Bates, D.J. Geolocation with FDOA Measurements via Polynomial Systems and RANSAC. In Proceedings of the 2018 IEEE Radar Conference (RadarConf18), Oklahoma City, OK, USA, 23-27 April 2018; pp. 0676-0681.

65. Rui, L.; Ho, K.C. Elliptic localization: Performance study and optimum receiver placement. IEEE Trans. Signal Process. 2014, 62, 4673-4688. [CrossRef]

66. Vesely, J.; Van Doan, S. Analytical Method Solving System of Hyperbolic Equations. In Proceedings of the 2015 25th International Conference Radioelektronika (RADIOELEKTRONIKA), Pardubice, Czech Republic, 21-22 April 2015; pp. 343-348.

67. Van Doan, S.; Vesely, J. The Effectivity Comparison of TDOA Analytical Solution Methods. In Proceedings of the 2015 16th International Radar Symposium (IRS), Dresden, Germany, 24-26 June 2015; pp. 800-805.

68. Van Doan, S.; Vesely, J.; Janu, P. The Measurement of TDOA Short Baseline. In Proceedings of the International Conference on Military Technologies (ICMT), Brno, Czech Republic, 19-21 May 2015; pp. 1-5.

69. Kuptsov, V.D.; Ivanov, S.I.; Fedotov, A.A.; Badenko, V.L. High-precision analytical TDoA positioning algorithm for eliminating the ambiguity of coordinates determination. IOP Conf. Ser. Mater. Sci. Eng. 2020, 904, 012013. [CrossRef]

70. Takahashi, Y.; Honma, N.; Sato, J.; Murakami, T.; Murata, K. Accuracy Comparison of Wireless Indoor Positioning Using Single Anchor: TOF only Versus TOF-DOA Hybrid Method. In Proceedings of the 2019 IEEE Asia-Pacific Microwave Conference (APMC), Singapore, 10-13 December 2019; pp. 1679-1681.

71. Vashist, A.; Bhanushali, D.R.; Relyea, R.; Hochgraf, C.; Ganguly, A.; Manoj, S.; Ptucha, R.; Kwasinski, A.; Kuhl, M.E. Indoor Wireless Localization Using Consumer-Grade $60 \mathrm{GHz}$ Equipment with Machine Learning for Intelligent Material Handling. In Proceedings of the 2020 IEEE International Conference on Consumer Electronics (ICCE), Las Vegas, NV, USA, 4-6 January 2020.

72. Piccinni, G.; Avitabile, G.; Coviello, G.; Talarico, C. Real-Time Distance Evaluation System for Wireless Localization. IEEE Trans. Circuits Syst. 2020, 1-11. [CrossRef]

73. Avitabile, G.; Florio, A.; Coviello, G. Angle of Arrival Estimation through a Full-Hardware Approach for Adaptive Beamforming. IEEE Trans. Circuits Syst. 2020, 1. [CrossRef]

74. Fokin, G.A.; Kireev, A.V.; Al-Odhari, A.H. TDOA Positioning Accuracy Performance Evaluation for Arc sensor Configuration. In Proceedings of the 2018 Systems of Signals Generating and Processing in the Field of on Board Communications, Moscow, Russia, 14-15 March 2018; pp. 1-5.

75. Liu, Y.; Yang, L.; Li, J. Robust UWB Indoor Position Tracking Using TDOA Measurements. In Proceedings of the 2018 IEEE 4th International Conference on Computer and Communications, Chengdu, China, 7-10 December 2018; pp. 736-743.

(C) 2020 by the authors. Licensee MDPI, Basel, Switzerland. This article is an open access article distributed under the terms and conditions of the Creative Commons Attribution (CC BY) license (http://creativecommons.org/licenses/by/4.0/). 